\title{
Threefold Increase in the Number of Drug Resistant TB Cases after Introduction of Universal Drug Susceptibility Testing: Experiences from Two South India Districts
}

\author{
Sanath Kumar Gurram Krishnamurthy ${ }^{1 *}$, Sharath Burugina Nagaraja ${ }^{2}$, Tanu Anand ${ }^{3}$, \\ Karuna D. Sagili ${ }^{4}$, Cheluve Gowda ${ }^{5}$, Shailaja ${ }^{6}$, Basavaraj Poojar ${ }^{7}$, Srinath Satyanarayana ${ }^{8}$ \\ ${ }^{1}$ Department of Health and Family, Tumkur District, Karnataka, India \\ ${ }^{2}$ Department of Community Medicine, ESIC Medical College and PGIMSR, Bengaluru, India \\ ${ }^{3}$ Department of Community Medicine, North Delhi Municipal Corporation Medical College, Hindu Rao Hospital, Delhi, India \\ ${ }^{4}$ Department of TB and Communicable Diseases, the Union South East Asia Office, New Delhi, India \\ ${ }^{5}$ Department of Community Medicine, Sri Siddhartha Medical College, Tumkur, Karnataka, India \\ ${ }^{6}$ Department of Health and Family, Belgaum District, Karnataka, India \\ ${ }^{7}$ Department of Pharmacology, Kasturba Medical College Mangalore, Manipal Academy of Higher Education, Manipal, India \\ ${ }^{8}$ The Union, South East Asia Office, New Delhi, India \\ Email: *dr.gurumsanath@gmail.com
}

How to cite this paper: Krishnamurthy, S.K.G., Nagaraja, S.B., Anand, T., Sagili, K.D., Gowda, C., Shailaja, Poojar, B. and Satyanarayana, S. (2020) Threefold Increase in the Number of Drug Resistant TB Cases after Introduction of Universal Drug Susceptibility Testing: Experiences from Two South India Districts. Journal of Tuberculosis Research, 8, 42-52.

https://doi.org/10.4236/jtr.2020.82005

Received: March 12, 2020

Accepted: May 24, 2020

Published: May 27, 2020

Copyright $\odot 2020$ by author(s) and Scientific Research Publishing Inc. This work is licensed under the Creative Commons Attribution International License (CC BY 4.0).

http://creativecommons.org/licenses/by/4.0/ (c) (i) Open Access

\begin{abstract}
Background: In India, tuberculosis (TB) is a major public health problem, and the advent of drug resistance TB (DR-TB) has worsened the situation. The Revised National TB Control Programme (RNTCP) has introduced universal drug susceptibility testing (UDST) for all diagnosed TB cases in 2018. We conducted this study to know the advantage of implementing UDST when compared to selective testing existent in 2017 on key diagnostic cascade parameters and to identify the challenges in the implementation of UDST. Methods: The study was conducted in two districts of Karnataka, India during January 2017-December 2018. The quantitative part consisted of before-and-after design and the qualitative part consisted of descriptive design. Results: In 2017 (during selective testing/"before" period) out of the $2440 \mathrm{~TB}$ patients, 80 (3\%) were diagnosed with Isoniazid and Rifampicin resistance patients; in contrast in 2018 (during UDST/"after" period) of the $5129 \mathrm{~TB}$ patients 258 (5\%) were diagnosed with Isoniazid and Rifampicin resistance. However, the proportion of eligible patients tested for rifampicin resistance during the "after" period was $60 \%$ when compared to $100 \%$ during the "before" period and median turnaround time for testing was also longer during the "after" period when compared to the "before" period (32.5 days vs 27.5 days).
\end{abstract}


Major reasons for these two gaps were found to be difficulties in collecting sputum specimens and transportation. Conclusion: The rollout of UDST has led to a three-fold increase in a number of DR-TB cases detected in the region. There is a need for the programme to increase the proportion tested for DST by increasing the laboratory capacity and address the challenges in sputum collection and transportation.

\section{Keywords}

Operational Research, Criteria C, Line Probe Assay, Universal Drug Susceptibility Testing, Culture and Drug Susceptibility Testing

\section{Introduction}

Globally, tuberculosis (TB) is a major public health problem and India accounts for more than $27 \%$ of the world's TB burden [1]. Worldwide, the estimated proportion of drug-resistant TB among new and retreatment cases is $4 \%$ and $19 \%$ respectively. And, there are an estimated 0.14 million drug-resistant tuberculosis (DR-TB) cases in India accounting for one-fourth of global TB burden [2].

To tackle the problem of drug resistance in TB, the Revised National TB Control Programme (RNTCP) of India initiated the programmatic management of drug-resistant TB (PMDT) in 2007. Initially, the programme was dependent on solid culture for drug susceptibility testing (DST), and there were a limited number of RNTCP accredited laboratories to perform the tests. Gradually, the programme expanded its horizon in a phased manner and included the line probe assay (LPA), liquid culture and cartridge-based nucleic acid amplification (CBNAAT or Xpert TB MTB/Rif) to detect DR-TB patients. By the end of 2018, the country had 74 Culture and drug susceptibility testing (CDST) laboratories, 106 LPA sites and 1135 CBNAAT sites across the country [3]. In view of identifying and diagnosing DR-TB patients the PMDT had laid down three different selective testing criteria namely A, B and C to prioritize presumptive DR-TB cases, keeping in consideration the availability of certified laboratories, necessary logistics and human resources [4].

The state of Karnataka in south India with 60 million population and 31 administrative districts was implementing criteria C/selective testing till 2017 [5]. The presumptive DR-TB cases in criteria $C$ included: 1) any sputum smear positive follow-up of new cases at the end of intensive phase, 2) all re-treated TB cases (sputum smear positive and negative) at the time of diagnosis, 3) all the contacts or presumptive TB cases of family to a known drug-resistant TB case and 4) All HIV-TB co-infected patients. During 2017, the state had 4 CDST laboratories, 4 LPA laboratories and 54 CB-NAAT sites delivering the services [3].

In January 2018, the state of Karnataka rolled out the policy of "universal drug susceptibility testing" (UDST) in which all the sputum smear-positive TB pa- 
tients will be subjected for DST. The intent of the initiative was to detect the drug-resistant TB patients early and reduce loss to follow-up and deaths. Implementation of the policy was a challenge to the programme staff and managers as it involved the transportation of sputum from designated microscopy centers in the field to the drug susceptibility testing (DST) sites which were located at far-away places.

We conducted this study in two districts of Karnataka, India to know the advantage of implementing UDST in 2018 ("after" period) when compared to the selective testing existent during 2017 ("before" period). We compared the key diagnostic cascade parameters that include the number (proportion) of eligible presumptive DR-TB cases, number (proportion) of eligible presumptive DR-TB tested, number (proportion) of DR-TB cases detected and drug resistance patterns, loss to follow-up and deaths and the median time taken for a presumptive TB case for DR-TB treatment initiation. We also explored the challenges in implementing UDST as perceived by the health care providers at these districts.

\section{Methods}

The quantitative part consisted of before-and-after design and the qualitative part consisted of descriptive design.

\subsection{Settings}

The study was conducted at two districts of Karnataka, namely Tumkur (2.9 million) and Belgaum (5.6 million) during June 2018 to April 2019 [5] (Figure 1). The districts were implementing PMDT since 2011. For the purpose of RNTCP implementation, the districts are further sub-divided into tuberculosis units (TU; for every 2.5 million population). The programme has established one designated microscopy centre (DMC) for every 0.1 million population where the laboratory technicians are trained for sputum smear examination and the laboratories are quality assured. The districts have primary health centres (PHC) for every 0.03 million population, and the DMCs are located at one of these PHCs. The district of Tumkur has 12 TUs with 34 DMCs and 3 CBNAAT sites while the district of Belgaum has 15 TUs with 49 DMCs and 5 CBNAAT sites spread across the vast geographical areas. During the initial years of PMDT implementation (2011-16), both the districts did not have any DST sites, and the samples were transported to the places outside the districts.

\subsection{PMDT Diagnostic Algorithms to Diagnose Drug Resistant TB} (Figure 2)

\section{Diagnostic cascade under selective testing (before)/Criteria C}

All the presumptive TB cases at DMCs would undergo sputum smear examination and those found to be positive are initiated on first-line anti-TB treatment. The patient is found to be sputum smear positive during a follow-up examination in the intensive phase are subjected to culture and DST. If found to be 
Map depicting study districts in state of Karnataka, India

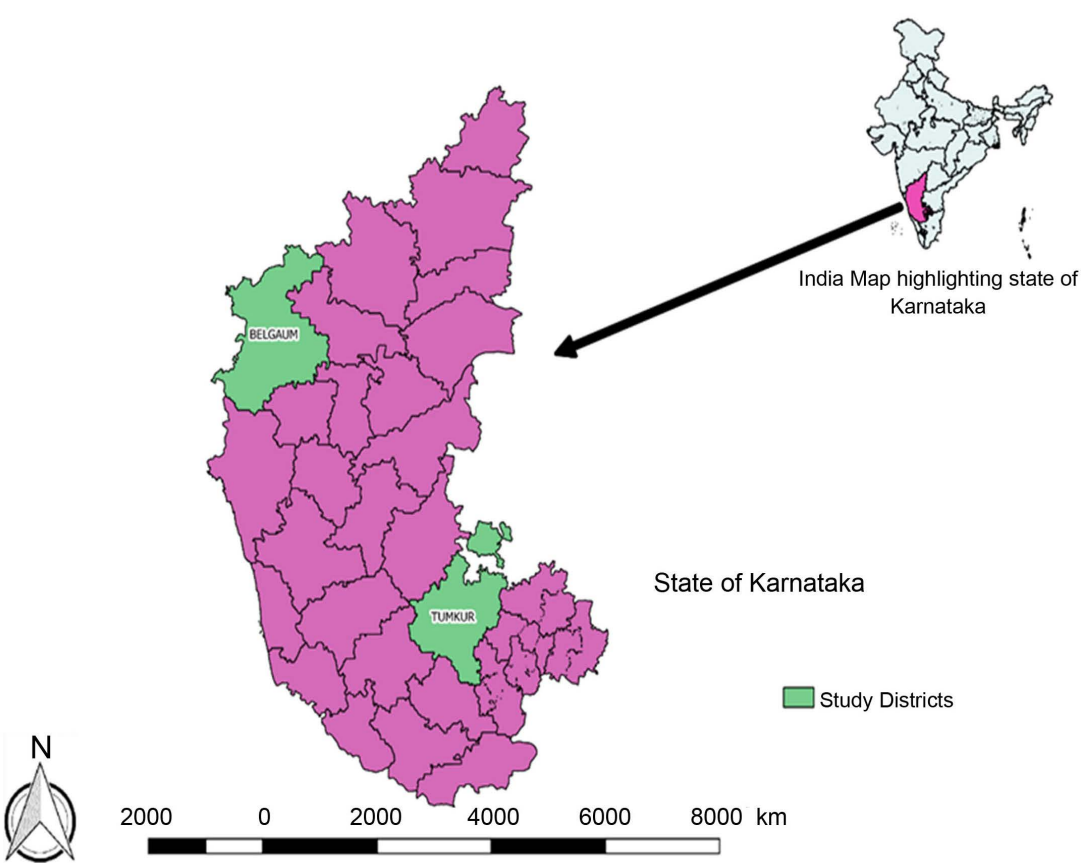

Figure 1. Map showing the location of districts chosen for the study.

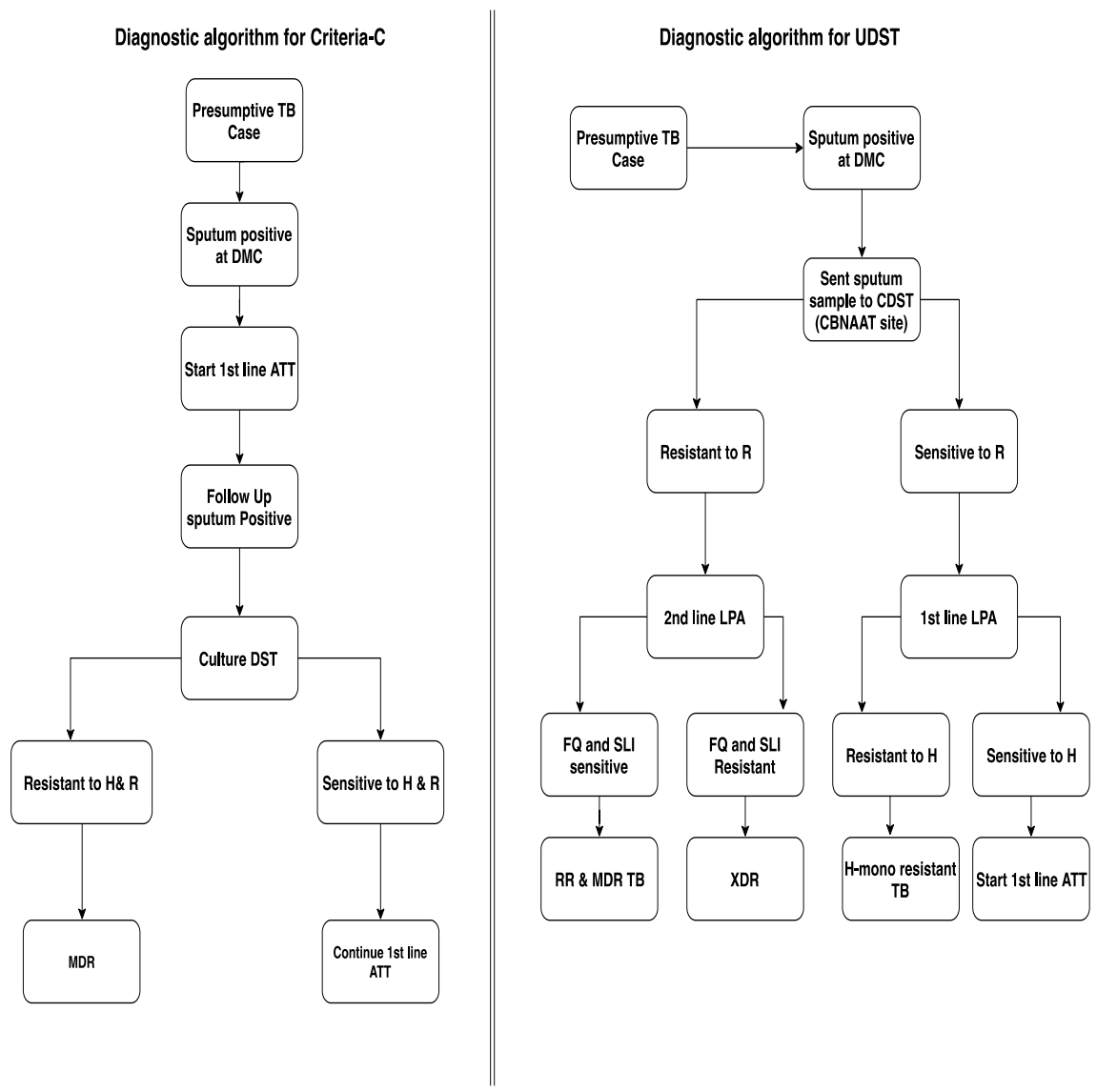

Figure 2. PMDT diagnostic algorithm for Criteria C (before) and UDST (after) under RNTCP. 
resistant to Isoniazid and Rifampicin, patients are initiated on multi-drug resistant TB (MDR-TB) treatment [6].

\section{Diagnostic cascade under Universal drug susceptibility testing (after)}

All the presumptive TB cases are subjected to sputum smear examination. If found positive, the sputum is sent to CBNAAT site for testing.

1) If the sputum is found to be sensitive to rifampicin (R), the sputum is subjected to first line LPA: a) If found to be sensitive to Isoniazid $(\mathrm{H})$, the patient is initiated on first line anti-TB treatment; b) If found to be resistant to Isoniazid, the patient is initiated on Isoniazid mono resistant TB.

2) If the sputum is found to be resistant to rifampicin, the sputum is subjected to second line LPA a) If found to be sensitive to fluoroquinolone (FQ) and second line (SLI) anti-TB drugs the patients are initiated on rifampicin resistant $\mathrm{TB}$ treatment $b$ ) If found to be resistant to FQ and SLI anti-TB drugs the patients are initiated on Extensively drug resistant TB (XDR-TB) treatment [6].

\section{Study Population, Sources of Data and Data Collection}

The quantitative data on presumptive DR-TB cases were obtained from the laboratory registers maintained at DMCs and the CDST registers at DST sites during January 2017 through December 2018. The qualitative data were obtained from the key informant interviews (KIIs) of 7 RNTCP staff of different cadres and 5 paramedical staff of different cadres involved in PMDT implementation. The KIIs were conducted at district TB centre in local vernacular language (Kannada) at a time and place convenient to them after taking prior appointment. Each interview lasted for $8-10$ minutes, and all the participants were purposively selected.

\subsection{Study Population, Sources of Data and Data Collection}

The quantitative data on presumptive DR-TB cases were obtained from the laboratory registers maintained at DMCs and the CDST registers at DST sites during January 2017 through December 2018. The qualitative data were obtained from the key informant interviews (KIIs) of 7 RNTCP staff of different cadres and 5 paramedical staff of different cadres involved in PMDT implementation. The KIIs were conducted at district TB centre in local vernacular language (Kannada) at a time and place convenient to them after taking prior appointment. Each interview lasted for 8 - 10 minutes, and all the participants were purposively selected.

\subsection{Data Variables}

Quantitative: The data on age, gender, sputum smear results, HIV status, date of diagnosis of drug sensitive TB, type of TB, type of TB treatment, date referred for DR-TB test, date of DR-TB results declared, date of pre-treatment evaluation and date of DR-TB treatment initiation were extracted from registers and reports. 
Qualitative: The challenges and suggested solutions were extracted from KII of general health care providers and RNTCP staff implementing UDST.

\subsection{Data Analysis}

The quantitative variables were double entered and validated using EpiData data entry software version 3.1. The data was analyzed using EpiData analysis (V2.2.2.2.182 Odense, Denmark). Descriptive statistics were computed on demographic variables. The proportions, median turnaround time and interquartile range were calculated. Chi-square test was used to detect any statistically significant difference and a p-value of less than 0.05 was considered to be significant. Manual descriptive thematic analysis was performed for qualitative data.

\subsection{Ethics Approval}

Ethics approval was obtained from the Institutional Ethics Committee of the ESIC Medical College and PGIMSR, Bangalore, Karnataka, India and the Ethics Advisory Group of the International Union against Tuberculosis and Lung Disease, Paris, France. For the quantitative component of the study, which involves the retrospective review of patient records, a waiver for informed consent was obtained from the ethics committees. Written informed consent were obtained from the stakeholder before interviews.

\section{Results}

\section{Quantitative}

The annual presumptive TB case load were 68735 and 71438 for the year 2017 and 2018. The comparison of the cascade of patients under selective testing (before) and UDST (after) is shown in Table 1. The sputum positivity rate among both the districts was found to be $10.7 \%$ both under selective testing and UDST. The proportion of death and loss to follow up before and during the first line anti-TB treatment were $16 \%$ and $12 \%$ respectively for selective testing and UDST. The proportion of patients who were eligible for testing under selective testing (before) were 33\% (2440/7386) and 100\% (7648/7648) for UDST (after).

The proportion of those tested were $100 \%$ (2440/2440) in selective testing and $67 \%$ (5129/7648) in UDST. Of those tested under selective testing (before), nearly $3.3 \%(80 / 2440)$ were found to be drug resistant (for isoniazid and rifampicin). Of those tested under UDST (after), nearly 5.03\% (258/5129) were found to be drug resistant (for isoniazid and rifampicin, isoniazid mono resistance and extensively drug resistance). Of the 258 found to be resistant in UDST (after), $60 \%$ (154) were isoniazid and rifampicin resistance, $32 \%$ (82) were isoniazid mono resistant and $8 \%$ (22) were extensively drug resistant TB. Nearly $20 \%$ (16; 8 died and $8 \mathrm{LFU}$ ) in selective testing (before) and 5.2\% (26; 11 died and $15 \mathrm{LFU})$ in UDST of diagnosed DR-TB patients were found to be dead or loss to follow up before initiation of treatment. The sociodemographic characteristics of diagnosed DR-TB patients under selective testing (before) and UDST are shown 
Table 1. Comparison of the cascade of tuberculosis patients during "criteria C/Selective testing (before)" and "UDST (after)" in Tumkur and Belgaum districts, Karnataka, 2017-18.

\begin{tabular}{ccc}
\hline Process & 2017 (Criteria C) & 2018 (UDST) \\
\hline $\begin{array}{c}\text { Number of presumptive TB cases tested } \\
\text { Number (\%) found to be smear positive }\end{array}$ & 68,735 & 71,438 \\
$\begin{array}{c}\text { Number (\%) of patients who were loss to follow-up } \\
\text { before and during first line anti-TB treatment } \\
\text { Number (\%) of patients who died before and } \\
\text { during first line anti-TB treatment }\end{array}$ & $566(7.6)$ & $7648(10.7)$ \\
$\begin{array}{c}\text { Number (\%) eligible for DR-TB testing } \\
\text { Number (\%) tested for DR-TB }\end{array}$ & $634(8.5)$ & $604(7.8)$ \\
Number found to be H + R resistant & $2440(33)$ & $7648(100)$ \\
$\begin{array}{c}\text { Number found to be H-mono resistant } \\
\text { Number found to be XDR }\end{array}$ & $2440(100)$ & $5129(67)$ \\
$\begin{array}{c}\text { Number (\%) of patients with any resistance } \\
\text { Number (\%) of patients who died } \\
\text { before DR-TB treatment initiation }\end{array}$ & $80(3.3)$ & $154(3.0)$ \\
$\begin{array}{c}\text { Number (\%) of patients who were LFU } \\
\text { before DR-TB treatment initiation }\end{array}$ & $80(3.27)$ & $22(1.5)$ \\
& $8(10)$ & $11(4.2)$ \\
& $8(10)$ & $15(1)$ \\
\hline
\end{tabular}

${ }^{*} \mathrm{p}$-value less than 0.05 .

in Table 2. Majority of the DR-TB patients were males in the age group of 26 to 55; there was no statistically significant difference between the two groups.

The median time required for a presumptive $\mathrm{TB}$ case to be initiated on DR-TB treatment under selective testing (before) was 27.5 days (IQR: 18 - 38.5) and 32.5 days (IQR: 9 - 59) under UDST (after).

\subsection{Qualitative}

The challenges perceived by the health care providers in implementation of UDST were broadly categorized as: 1) collection and transportation of samples and 2) costs for transportation.

\subsection{Collection and Transportation of Sputum Samples}

The health care providers opined that tracing the presumptive TB case who is found to be smear positive TB for drug sensitive TB is a difficult task. The patient either comes to collect the sputum results next day or the health care provider informs the paramedical staff of the area through WhatsApp (social media mobile application freely available) or phone call. The provider also has to visit the house of the patient to collect the sample and send it to DMC. The cumulatively collected such sputum containers are transported to the DST site once in two days either by a courier or a human carrier. The time taken to transport sample from field to CBNAAT site and then to LPA testing at DST laboratory is 7 - 10 days. Many a times the sputum samples are inappropriately packed and leads to spillage. Hence, the providers are reluctant to transport the samples. 
Table 2. Socio-demographic characteristics of diagnosed DR-TB patients under criteria C/selective testing (before) and UDST (after), in Tumkur and Belgaum districts, 2017-18.

\begin{tabular}{|c|c|c|}
\hline Variables & $\begin{array}{c}\text { Criteria C } \\
\text { n (\%) }\end{array}$ & $\begin{array}{l}\text { UDST } \\
\text { n (\%) }\end{array}$ \\
\hline \multicolumn{3}{|l|}{ Age } \\
\hline $0-15$ & $3(4)$ & $4(2)$ \\
\hline $16-25$ & $16(20)$ & $41(16)$ \\
\hline $26-35$ & $21(26)$ & $45(17)$ \\
\hline $36-45$ & $20(25)$ & $63(24)$ \\
\hline $46-55$ & $9(11)$ & $53(20)$ \\
\hline $56-65$ & $8(10)$ & $33(12.8)$ \\
\hline 65 and above & $3(4)$ & $19(7.4)$ \\
\hline \multicolumn{3}{|l|}{ Gender } \\
\hline Male & $53(66)$ & $184(72)$ \\
\hline Female & $27(34)$ & $73(28)$ \\
\hline Not recorded & $0(0)$ & $01(4)$ \\
\hline \multicolumn{3}{|l|}{ Sputum Results } \\
\hline Positive & $66(82.5)$ & $248(96.5)$ \\
\hline Negative & $07(8.8)$ & $9(3.5)$ \\
\hline Not recorded & $07(8.8)$ & $0(0)$ \\
\hline \multicolumn{3}{|l|}{ Type of TB } \\
\hline Pulmonary & $78(97.5)$ & $255(98.8)$ \\
\hline Extra-pulmonary & $02(2.5)$ & $3(1.2)$ \\
\hline \multicolumn{3}{|l|}{ Type of TB Treatment } \\
\hline Newly detected & $33(41.3)$ & $94(36.4)$ \\
\hline Previously Treated & $47(58.8)$ & $164(63.6)$ \\
\hline \multicolumn{3}{|l|}{ HIV } \\
\hline Negative & $76(95)$ & $256(99.2)$ \\
\hline Positive & $4(5)$ & $2(0.8)$ \\
\hline
\end{tabular}

\section{Costs of transportation}

The providers incur the costs of at least INR 20 - 50 to visit the patients for collection of sputum which is usually not reimbursed by the peripheral health centers; and to avoid this cost, the providers insist on patients to provide the sputum samples at DMC. This enforcement on patients indirectly affects the daily earnings of the patients.

\section{Discussion}

It is the first study conducted in the country to know the benefits of UDST implementation. The study findings suggest that the roll out of UDST has led to three-fold increase in number of DR-TB cases detected in the region. The im- 
portant challenge perceived by health care providers in implementation of UDST is collection and transportation of sputum samples to DMCs.

The study has following programmatic implications. First, under UDST (after) only $67 \%$ of TB patients were tested for DST when compared to $100 \%$ eligible patients under selective testing (before). The pre-diagnosis attrition for UDST was $67 \%$ in this study when compared to $88 \%$ and $60 \%$ among studies conducted at Chennai and Bhopal in India for DST [7] [8]. The probable reason for this attrition in the study could be due to delay in patient's sputum sample collection and transportation to DMC and due to attrition from loss to follow-up and deaths; which were in contrast to non-identification of eligible presumptive DR-TB cases in a study conducted at Bhopal [9]. The 258 DR-TB cases detected under UDST could be an underestimate as not all eligible were tested and we presume that an additional of 100 DR-TB cases could have been detected if all the diagnosed patients were to be tested for DST.

Second, the UDST has the additional capability of detecting Isoniazid mono resistant and XDR-TB which otherwise would have not been detected under selective testing (before). These XDR-TB cases were detected among the new cases examined at DMCs and thus avoids the churning of patients in the health system. Studies have shown that pre-treatment mortality gets reduced on testing the patients early and programs should encourage early detection. [10] [11] further operational research studies are needed to find out the treatment outcomes and survival rates of those DR-TB patients detected under selective testing (before) and UDST (after) in India.

Third, the median turnaround time from being a presumptive TB case to initiation DR-TB treatment was 27.5 days in selective testing (before) and 32.5 days in UDST (after). A systematic review suggests that the time to treatment initiation for second line TB treatment is 38 days (95\% CI 27 - 49) for genotypic susceptibility testing [12]. The reason for longer duration in UDST could be related to: 1) delay in collection of sputum from patient site and sending it to DMC;2) flooding of sputum samples from peripheries to CBNAAT site at the district; 3) overburdening of DST sites by influx of samples from the catchment area of zonal districts. The programme should consider destressing the laboratories by placing CBNAAT machines at sub-district levels and providing LPA sites at district level.

Fourth, there remains a challenge of sputum transportation at sub-district level. Innovative strategies need to be developed and the non-governmental organizations are to be encouraged to participate by providing attractive incentives. Mechanisms have to be developed to re-imburse the cost incurred for sputum transportation by the health providers at the level of peripheral health centers.

The study has following strengths and limitations. The strengths are: 1) the study is conducted under field conditions and hence reflects the ground reality; 2) the data is considered verified as the quantitative data was double-entered and validated for discrepancies; 3 ) the qualitative component of the study has helped in identifying the challenges pertaining to diagnosis. The limitations are: 1) there 
were few missing and incomplete data which is considered to be normal for quantitative studies under programmatic conditions; 2) the interviewer for the key informants of Belgaum district was the district TB officer from Tumkur district and there could be a possibility of introducing bias among the interviewees; 3) the findings of the study is contextual to this situation and should be generalized to other regions with caution.

To conclude, the rollout of UDST has led to a three-fold increase in a number of DR-TB cases detected in the region. There is a need for the programme to increase the proportion tested for DST by increasing the laboratory capacity and address the challenges in sputum collection and transportation.

\section{Acknowledgements}

This research was conducted as a part of the "National Operational Research Training Course 2018-19" organised by Project Axshya, funded by the Global Fund and implemented by The International Union Against Tuberculosis and Lung Diseases (The Union), South-East Asia Regional Office, New Delhi, India. The training course was conducted in collaboration with Revised National Tuberculosis Control Program, Ministry of Health and Family Welfare, Government of India and National Institute for TB and Respiratory Diseases, New Delhi, India.

The training is based on "The Union/Medécins sans Frontières (MSF)" model OR course and which has been adapted as SORT IT (Structured Operational Research and Training Initiative).coordinated by TDR, the Special Programme for Research and Training in Tropical Diseases at the World Health Organization Mentorship and facilitation for this course was provided through the Union South-East Asia Office, New Delhi; the Centre for Operational Research, The Union, Paris, France; ESIC Medical College and PGIMSR, Bengaluru; Baroda Medical College, Vadodara; Médecins Sans Frontières, New Delhi; North Delhi Municipal Corporation Medical College, Hindu Rao Hospital, New Delhi; GMERS Medical College, Vadodara; Postgraduate Institute of Medical Education and Research, Chandigarh, India; Yenepoya Medical College, Mangalore.

\section{Funding}

The training course under which this research was conducted was funded by The Global Fund to Fight AIDS, Tuberculosis and Malaria (GFATM). The funders had no role in study design, data collection and analysis, decision to publish, or preparation of the manuscript. Open access publication costs were covered by TDR.

\section{Conflicts of Interest}

The authors declare no conflicts of interest regarding the publication of this paper.

\section{References}

[1] World Health Organization (2018) Global WHO Report on Tuberculosis 2018. Geneva, Switzerland, Switzerland. 
[2] Directorate of General Health Services (2017) First National Anti-Tuberculosis Drug Resistance Survey India 2014-16.

[3] Central TB Division (2018) TB India Report 2018. https://tbcindia.gov.in/showfile.php?lid=3314

[4] Dye, C. and Williams, B.G. (2002) Criteria for the Control of Drug-Resistant Tuberculosis. Proceedings of the National Academy of Sciences, 97, 8180-8185. https://doi.org/10.1073/pnas.140102797

[5] Census (2019) List of districts of Karnataka. https://www.census2011.co.in/census/state/districtlist/karnataka.html

[6] Central TB Division (2017) Guidelines on Programatic Management of Drug Resistant Tuberculosis in India 2017. Central TB Division, New Delhi, India. https://tbcindia.gov.in/index1.php?lang=1\&level=2\&sublinkid=4780\&lid=3306

[7] Shewade, H.D., Kokane, A.M., Singh, A.R., Verma, M., Parmar, M., Chauhan, A., et al. (2017) High Pre-Diagnosis Attrition among Patients with Presumptive MDR-TB: An Operational Research from Bhopal District, India. BMC Health Services Research, 17, 249. https://doi.org/10.1186/s12913-017-2191-6

[8] Shewade, H.D., Nair, D., Klinton, J.S., Parmar, M., Lavanya, J., Murali, L., et al. (2017) Low Pre-Diagnosis Attrition But High Pre-Treatment Attrition among Patients with MDR-TB: An Operational Research from Chennai, India. Journal of Epidemiology and Global Health, 7, 227-233.

https://doi.org/10.1016/j.jegh.2017.07.001

[9] Shewade, H.D., Kokane, A.M., Singh, A.R., Parmar, M., Verma, M., Desikan, P., et al. (2018) Provider Reported Barriers and Solutions to Improve Testing among Tuberculosis Patients "Eligible for Drug Susceptibility Test”: A Qualitative Study from Programmatic Setting in India. PLOS ONE, 13, e 0196162. https://doi.org/10.1371/journal.pone.0196162

[10] Evans, D., Sineke, T., Schnippel, K., Berhanu, R., Govathson, C., Black, A., et al. (2018) Impact of Xpert MTB/RIF and Decentralized Care on Linkage to Care and Drug-Resistant Tuberculosis Treatment Outcomes in Johannesburg, South Africa. BMC Health Services Research, 18, 973. https://doi.org/10.1186/s12913-018-3762-x

[11] Harris, R.C., Grandjean, L., Martin, L.J., Miller, A.J.P., Nkang, J.-E.N., Allen, V., et al. (2016) The Effect of Early versus Late Treatment Initiation after Diagnosis on the Outcomes of Patients Treated for Multidrug-Resistant Tuberculosis: A Systematic Review. BMC Infectious Diseases, 16, 193. https://doi.org/10.1186/s12879-016-1524-0

[12] Boyd, R., Ford, N., Padgen, P. and Cox, H. (2017) Time to Treatment for Rifampicin-Resistant Tuberculosis: Systematic Review and Meta-Analysis. The international Journal of Tuberculosis and Lung Disease: The Official Journal of the International Union against Tuberculosis and Lung Disease, 21, 1173-1180.

https://doi.org/10.5588/ijtld.17.0230 